\title{
Exponentially decaying correlations in a gas of strongly interacting spin-polarized 1D fermions with zero-range interactions
}

\author{
Scott A. Bender, Kevin D. Erker, and Brian E. Granger \\ Department of Physics, Santa Clara University, Santa Clara, CA 95053
}

\begin{abstract}
We consider the single particle correlations and momentum distributions in a gas of strongly interacting spinless 1D fermions with zero-range interactions. This system represents a fermionic version of the TonksGirardeau gas of impenetrable bosons as it can be mapped to a system of noninteracting 1D bosons. We use this duality to show that the $T=0$ single particle correlations exhibit an exponential decay with distance. This strongly interacting system is experimentally accessible using ultracold atoms and has a Lorentzian momentum distribution at large momentum whose width is given by the linear density.

PACS numbers: 03.75.-b, 03.75.Ss, 05.30.Fk
\end{abstract}

One dimensional (1D) many body systems provide a beautiful example of duality. In this context, duality refers to the precise mappings that exist between weakly interacting $1 \mathrm{D}$ bosons and strongly interacting $1 \mathrm{D}$ fermions and vice versa. A number of classic examples of duality in 1D systems exist: i) the Luttinger liquid [1, 2] which is dual to a collection of noninteracting bosonic collective modes, ii) the Tonks-Girardeau gas of impenetrable bosons [3, 4], which is dual to a noninteracting gas of fermions and iii) the quantum sine-Gordon model, which is dual to the massive Thirring model [5].

More recently, a number of groups [6, 7, 8, 9, 10] have introduced equivalent models of spinless $1 \mathrm{D}$ fermions with zero-range interactions. Again, duality appears in these models as they can all be mapped to a system of $\delta$-function interacting 1D bosons. These models contain the familiar bosonic Tonks-Girardeau gas [3, 4] as a specific case as well as a fermionic version of the Tonks-Girardeau (TG) gas [8, 9] in which strongly interacting fermions are related to noninteracting bosons. In this paper we discuss the correlations among these 1D fermions with strong interactions.

Ultracold atom gases provide an excellent arena in which to study 1D many body systems with short-range interactions. Already, ultracold atoms have been confined in quasi1D (highly elongated) harmonic traps [11] where phase fluctuations in quasi-1D Bose-Einstein condensates [12, 13] and the TG gas [14, 15] have been observed.

Although such experiments are actually three dimensional (3D) in nature, their low energy properties can be modeled by an effective 1D Hamiltonian, as first demonstrated by Olshanii [16]. For quasi-1D bosons, this model consists of mass $m 1 \mathrm{D}$ bosons interacting through the potential $V(z)=g_{1 D}^{B} \delta(z)$, where the interaction strength, $g_{1 D}^{B}$, is chosen to correctly reproduce the low energy behavior of the full 3D Hamiltonian of the harmonically confined atoms. Most importantly, $g_{1 D}^{B}$ can be tuned to any value (even infinite) by changing ratio $a_{s} / a_{\perp}$, where $a_{s}$ is the 3D $s$-wave scattering length of the bosons and $a_{\perp}$ is the length scale of the tight harmonic confinement. Thus, ultracold atoms in quasi-1D traps can be used to probe the well studied uniform 1D Bose gas with $\delta$-function interactions [17, 18, 19]. The dimensionless interaction strength for this system is $\gamma_{B}=\frac{m g_{1 D}^{B}}{n \hbar^{2}}$, where $n$ is the linear density. In the strongly interacting limit $\left(\gamma_{B} \rightarrow \infty\right)$ the $\delta$-interacting 1D Bose gas reduces to the recently observed TG gas $[12,15,16]$.
Of critical importance in these studies are the static correlation functions. These determine experimentally measurable properties such as momentum distributions [14] and inelastic collision rates [20]. For the $\delta$-interacting Bose gas, these correlation functions are known in a number of different regimes. In the TG regime $\left(\gamma_{B} \rightarrow \infty\right)$ duality can be used to calculate the single particle correlations for all densities and distances [16, 21]. For finite interactions $\gamma_{B}$ the single- and local multi- particle correlation functions are known analytically in a number of limits (see [22, 23, 24] and the references therein). Lastly, numerical calculations have covered all interaction strengths for both uniform and harmonically confined systems [25]. The most important feature in all these studies is that single particle correlations at zero temperature $(T=0)$ exhibit a power law decay with distance. This power law decay of correlations is also shared by the classic Luttinger liquid model of spinless 1D fermions [26].

With this background we turn our attention to the single particle correlations in the recently proposed model of spinless 1D fermions with zero-range interactions. Our focus is upon strongly interacting 1D fermions, which are dual to noninteracting bosons; a sort of fermionic version of the TG gas. We emphasize that this system does not appear to be another obvious manifestation of the Luttinger liquid. This becomes evident in the nature of the single particle correlations, which we find decay exponentially with distance at $T=0$.

Our method consists of using duality to obtain a general relationship between the single particle density matrices of the fermions and their dual bosons. Thus, once the single particle correlations are known for noninteracting bosons in any external potential, the fermionic correlations are known immediately. We apply these general results to uniform and harmonically confined 1D gases, and give the experimentally relevant momentum distributions for each.

Spin polarized fermions. Here we introduce and summarize the main features of the 1D spinless fermions considered in this paper. This model has been introduced by a number of researchers in different forms [6, 7, 8, 9, 10]. Consider a Hamiltonian for $N$ mass $m$ 1D spinless fermions,

$$
H_{F}=\frac{-\hbar^{2}}{2 m} \sum_{i=1}^{N} \frac{\partial^{2}}{\partial z_{i}^{2}}+\sum_{i<j}^{N} V_{F}\left(\left|z_{i}-z_{j}\right|\right)
$$

with a zero-range interaction potential $V_{F}(z)$ that has a non- 
trivial action on the space of antisymmetric wavefunctions $\psi_{F}\left(x_{1}, \ldots, x_{N}\right)$. The zero-range interaction potential $V_{F}(z)$, parameterized by an interaction strength $g_{1 D}^{F}$, can be chosen in a number of ways, including [6]

$$
V_{F}(z)=\lim _{s \rightarrow 0} \frac{-\hbar^{2}}{2 \mu}\left(\frac{\hbar^{2}}{\mu g_{1 D}^{F}}+\frac{1}{s}\right)[\delta(z-s)+\delta(z+s)]
$$

and [27]

$$
V_{F}(z)=g_{1 D}^{F} \frac{\overleftarrow{\partial}}{\partial z} \delta(z) \frac{\vec{\partial}}{\partial z}
$$

At first glance, it is not obvious that these and the other [7, 9, 10, 28] zero-range potentials for 1D spinless fermions are equivalent. However, when treated exactly, they all lead to the same logarithmic derivative of the relative wavefunction $\psi_{\text {rel }}(z)$ where two particles touch ( $z$ is the distance between the particles) [6]:

$$
\left[\frac{d \psi_{\text {rel }}(z)}{d z} \frac{1}{\psi_{\text {rel }}(z)}\right]_{z \rightarrow 0^{+}}=-\frac{\hbar^{2}}{\mu g_{1 D}^{F}} .
$$

The dimensionless interaction strength for this model is $\gamma_{F}=$ $\frac{m g_{1 D}^{F}}{\hbar^{2}}$ so that weak interactions $\left(\gamma_{F} \ll 1\right)$ occur for low density and small $g_{1 D}^{F}$ and strong interactions $\left(\gamma_{F} \gg 1\right)$ occur at high density and large $g_{1 D}^{F}$.

Recently, one of us (B. Granger), in collaboration with D. Blume, showed that this model of 1D fermions with zero-range interactions describes the physics of spin-polarized fermionic atoms in quasi-1D harmonic traps at ultracold temperatures [8]. Then, the effective 1D interaction strength $g_{1 D}^{F}$ depends on the ratio $V_{p} / a_{\perp}^{3}$, where $V_{p}$ is the $p$-wave scattering volume of the atoms and $a_{\perp}$ the length scale of the tight harmonic confinement. Thus, by changing either $V_{p}$ (using a Feshbach resonance [29]) or $a_{\perp}$ (by modifying the trap) $g_{1 D}^{F}$ can be tuned over a large range of values, including infinity. Thus, it should be possible to study strongly interacting 1D fermions using ultracold atoms; just as strongly interacting 1D bosons have been studied in recent experiments [14, 15].

To calculate the single particle correlations in the strongly interacting limit $\left(\gamma_{F} \rightarrow \infty\right)$ we map the 1D fermions to a system of 1D bosons with reciprocal interaction strength. This mapping was first used by Girardeau [4] to relate impenetrable bosons to noninteracting fermions. Recently, the mapping has been extended to relate 1D fermions with any interaction strength to $1 \mathrm{D}$ bosons with $\delta$-function interactions [6, 7, 8, 9, 10]. More specifically, any antisymmetric wavefunction $\psi_{F}\left(z_{1}, \ldots, z_{N}\right)$ for $N$ 1D fermions with zero-range interactions of strength $g_{1 D}^{F}$ is related to a symmetric wavefunction $\psi_{B}\left(z_{1}, \ldots, z_{N}\right)$ of $N 1 \mathrm{D}$ bosons with $\delta$-function interactions of strength $g_{1 D}^{B}$ through the mapping [4]:

$$
\psi_{F}\left(z_{1}, \ldots, z_{N}\right)=\left[\prod_{j<k}^{k=N} \operatorname{sgn}\left(z_{j}-z_{k}\right)\right] \psi_{B}\left(z_{1}, \ldots, z_{N}\right) .
$$

When the interaction strengths of the bosons and fermions are chosen to be reciprocals of each other,

$$
g_{1 D}^{F}=\frac{-\hbar^{4}}{\mu^{2} g_{1 D}^{B}},
$$

the energy spectrum, thermodynamic properties and local correlations (as $\left|\prod_{j<k}^{k=N} \operatorname{sgn}\left(z_{j}-z_{k}\right)\right|^{2}=1$ ) of the two systems are identical. Thus, for example, strongly interacting fermions $\left(\gamma_{F} \rightarrow \infty\right)$ share these properties with the noninteracting 1D Bose gas.

Not all properties, however, are shared by the fermions and their bosonic partners. In fact, any nonlocal correlation function will be different. Thus, nonlocal correlations provide an important and unique signature of the actual exchange statistics of the physical particles. We now derive the single particle correlations and momentum distributions for the 1D fermions described above in the strongly interacting limit $\left(\gamma_{F} \rightarrow \infty\right)$.

General form of the density matrix. For both bosons and fermions, the single particle reduced density matrix is defined as

$$
\begin{aligned}
\rho\left(z, z^{\prime}\right) & =N \int_{a}^{b} \psi\left(z, z_{2}, \ldots, z_{N}\right) \psi^{*}\left(z^{\prime}, z_{2}, \ldots, z_{N}\right) \\
& \times d z_{2} \ldots d z_{N},
\end{aligned}
$$

so that $\operatorname{Tr}[\rho]=N$. The ground state wavefunction, $\psi_{B}$, for $N$ noninteracting bosons has the familiar form

$$
\psi_{B}\left(z_{1}, \ldots, z_{N}\right)=\prod_{i=1}^{N} \phi_{0}\left(z_{i}\right),
$$

where $\phi_{0}\left(z_{i}\right)$ is the normalized ground state solution to the single particle Schrödinger equation in the region $a \leq z \leq b$. From the form of $\psi_{B}$ in Eq. [8, the reduced density matrix for noninteracting bosons, $\rho_{B}\left(z, z^{\prime}\right)$, is easily found and quite simple:

$$
\rho_{B}\left(z, z^{\prime}\right)=N \phi_{0}(z) \phi_{0}^{*}\left(z^{\prime}\right) .
$$

Finding the density matrix, $\rho_{F}\left(z, z^{\prime}\right)$, for $1 \mathrm{D}$ fermions with strong $\left(\gamma_{F} \rightarrow \infty\right)$ zero-range interactions, on the other hand, is nontrivial, but can be accomplished by using duality. We construct the interacting fermion wavefunction in the $\gamma_{F} \rightarrow \infty$ limit using the noninteracting boson wavefunction, Eq. 8, and the mapping, Eq. (5). Then, substitution of the fermion wave function $\psi_{F}$, Eq. (5), into the definition of the density matrix, Eq. (7), yields an integral expression for the single particle fermionic density matrix in terms of the orbitals of the noninteracting bosons:

$$
\begin{aligned}
\rho_{F}\left(z, z^{\prime}\right) & =N \phi_{0}(z) \phi_{0}^{*}\left(z^{\prime}\right) \int_{a}^{b}\left(\prod_{i=2}^{N} \operatorname{sgn}\left(z-z_{i}\right) \operatorname{sgn}\left(z^{\prime}-z_{i}\right)\right) \\
& \times\left(\prod_{1<j<k}^{k=N}\left(\operatorname{sgn}\left(z_{j}-z_{k}\right)\right)^{2} \phi_{0}\left(z_{k}\right) \phi_{0}^{*}\left(z_{k}\right)\right) \\
& \times d z_{2} \ldots d z_{N} .
\end{aligned}
$$

Noticing that $\left(\operatorname{sgn}\left(z_{j}-z_{k}\right)\right)^{2}=1$ in this expression, the multidimensional integrals can be separated into a product of $N-1$ 
integrals over each $d z_{i}$. A careful treatment of these integrals gives the final expression for the single particle reduced density matrix for 1D fermions in the strongly interacting $\left(\gamma_{F} \rightarrow \infty\right)$ limit:

$$
\rho_{F}\left(z, z^{\prime}\right)=\rho_{B}\left(z, z^{\prime}\right)\left(1-2 P\left(z, z^{\prime}\right)\right)^{N-1} .
$$

In this expression

$$
P\left(z, z^{\prime}\right)=\frac{1}{N}\left|\int_{z}^{z^{\prime}} \rho_{B}\left(z^{\prime \prime}, z^{\prime \prime}\right) d z^{\prime \prime}\right|
$$

is the probability of finding a noninteracting boson between $z$ and $z^{\prime}$. This result gives a completely general relationship between the single particle density matrices of noninteracting bosons and strongly interacting fermions in 1D.

In many cases it is possible to take the thermodynamic limit of these results $(N \rightarrow \infty, L \rightarrow \infty$ but $n=N / L$ fixed, where $L$ is some characteristic length for the system). If $\tilde{P}\left(z, z^{\prime}\right)=$ $L P\left(z, z^{\prime}\right)$ is independent of $N$ and $L$, then the expression

$$
e^{x}=\lim _{N \rightarrow \infty}(1+x / N)^{N}
$$

can be used to take the thermodynamic limit of the fermion density matrix, Eq. (11):

$$
\rho_{F}=\rho_{B} e^{-2 n \tilde{P}\left(z, z^{\prime}\right)} .
$$

Typically $\tilde{P}\left(z, z^{\prime}\right) \sim\left|z-z^{\prime}\right|$, so that the 1D fermions generally exhibit an exponential decay of correlations with distance compared to noninteracting bosons.

Periodic system. Now that we have the general expression, Eqs. (11) and (12), for the single particle density matrix in the limit $\gamma_{F} \rightarrow \infty$, we can apply this result to specific external potentials. First, consider a constant potential with periodic boundary conditions over the interval $[0, L]$. The ground state solution for each orbital is just $\phi_{0}\left(z_{j}\right)=\sqrt{\frac{1}{L}}$, so from Eq. (9), $\rho_{B}=n$. The fermion density matrix then follows from Eqs. (11) and (12):

$$
\rho_{F}\left(z, z^{\prime}\right)=n\left(1-2\left|\frac{z^{\prime}-z}{L}\right|\right)^{N-1} .
$$

The thermodynamic limit $(N \rightarrow \infty, L \rightarrow \infty$ and $n=N / L$ fixed) of this result,

$$
\rho_{F}\left(z, z^{\prime}\right)=n e^{-2 n\left|z^{\prime}-z\right|},
$$

exhibits the promised exponential decay with distance. Alternatively, the single particle correlations can be characterized by the correlation function, $g_{1}(z)=\rho_{F}(z, 0) / n=$ $\exp (-2 n|z|)$, which is normalized as $g_{1}(0)=1$. This exponentially decaying single particle correlation function is one of the central results of this paper and clearly shows the striking effect that the boson $\rightarrow$ fermion mapping, Eq. (5), has on the nature of single particle correlations. More specifically, the long-range order of $T=0$, noninteracting $1 \mathrm{D}$ bosons becomes an exponential decay of correlations (with correlation length $\xi=\frac{1}{2 n}$ ) in the strongly interacting $1 \mathrm{D}$ fermions considered here. Furthermore, this behavior is a dramatic departure from the power law decay of correlations exhibited by the $T=0 \delta$-interacting 1D Bose gas and Luttinger liquid.

Of particular importance for experiments with ultracold atoms is the momentum distribution $n(k)$, which is obtained as the Fourier transform of the correlation function $g_{1}(z)$ :

$$
\begin{aligned}
n(k) & =\frac{1}{2 \pi} \int_{-\infty}^{\infty} e^{i k z} g_{1 F}(z) d z \\
& =\frac{2 n}{\pi\left(4 n^{2}+k^{2}\right)} .
\end{aligned}
$$

This Lorentzian momentum distribution shows the signature effect of strong interactions in a system of zero-temperature fermions. That is, interactions have completely smoothed out the sharp decrease of the momentum distribution at the Fermi momentum $k_{F} \approx 2 \pi n$.

It is also interesting to note that the width of this momentum distribution is given by the linear density $n$. As $n \rightarrow 0$ this momentum distribution becomes identical to that of a noninteracting gas of zero-temperature 1D bosons, $\delta(k)$. This is quite an unexpected momentum distribution for a strongly interacting gas of fermions. While it is tempting to speculate about the physical meaning of this feature (is it a noninteracting Bose gas of molecules?), we warn that our results are only relevant in the high density limit $\left(\gamma_{F} \rightarrow \infty\right)$ limit.

Harmonic confinement. Finally, we can determine the density matrix and momentum distribution for $N$ 1D fermions of mass $m$ confined to a harmonic oscillator of frequency $\omega_{z}$ in the $z$ direction. The normalized orbitals are

$$
\phi_{0}\left(z_{j}\right)=\sqrt{\frac{1}{a_{z} \sqrt{\pi}}} e^{-z_{j}^{2} / 2 a_{z}^{2}},
$$

where $a_{z}=\sqrt{\hbar / m \omega_{z}}$. The density matrix for noninteracting $1 \mathrm{D}$ bosons, Eq. (9), is then

$$
\rho_{B}=n e^{\frac{-z^{2}-z^{2}}{2 a_{z}^{2}}},
$$

where we define the linear density for the harmonic oscillator as $n=\frac{N}{a_{z} \sqrt{\pi}}$. The density matrix for our strongly interacting 1D fermions, Eqs. (11) and (12), can be written in terms of $\operatorname{Erf}(x)=\frac{2}{\sqrt{\pi}} \int_{0}^{x} e^{-t^{2}} d t$ :

$$
\rho_{F}\left(z, z^{\prime}\right)=\rho_{B}\left(1-\left|\operatorname{Erf}\left(\frac{z}{a_{z}}\right)-\operatorname{Erf}\left(\frac{z^{\prime}}{a_{z}}\right)\right|\right)^{N-1} .
$$

From this expression, we calculate the momentum distribution $n(k)$ numerically, by Fourier transforming the density matrix,

$$
\rho_{F}\left(k, k^{\prime}\right)=\frac{1}{2 \pi} \int_{-\infty}^{\infty} \int_{-\infty}^{\infty} e^{i k z} e^{-i k^{\prime} z^{\prime}} \rho\left(z, z^{\prime}\right) d z d z^{\prime},
$$

so that $n(k)=\rho_{F}(k, k)$.

Figure 1 shows the calculated momentum distributions of harmonically confined, strongly interacting 1D fermions for $N=5,20$ and 100 particles. The main figure shows clearly 


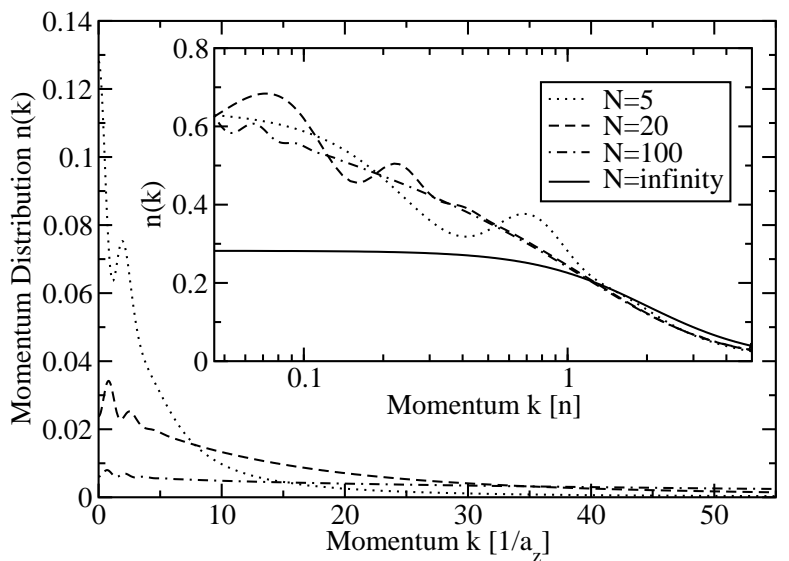

Figure 1: Momentum distributions $n(k)$ are shown as a function of $k$ for a 1D gas of strongly interacting spinless fermions with 1D harmonic confinement of frequency $\omega_{z}$. The main figure shows the momentum distributions, normalized to unity with the momentum given in units of $1 / a_{z}$. The inset shows the momentum distribution (normalized to $N$ ) with the momentum given in units of the density $n=N / a_{z} \sqrt{\pi}$. For the $N=\infty$ case we have plotted the Lorentzian, Eq. 18, that corresponds to the thermodynamic limit.

that the widths of these distributions increase in proportion with the linear density $n$. This is the opposite behavior from what has been calculated for harmonically confined 1D interacting bosons [25]. The oscillations at small momentum are due to the harmonic confinement rather than the details of the interactions.

At a more quantitative level, the large momentum tails of these distributions can be understood by taking the thermodynamic limit of the density matrix, Eq. 21. This limit is specified by letting $a_{z} \sqrt{\pi} \rightarrow \infty$ and $N \rightarrow \infty$ with $n=N / a_{z} \sqrt{\pi}$ fixed. In this limit, we can use the small argument approximation $\operatorname{Erf}\left(\frac{z}{a}\right) \approx \frac{2 z}{a_{z} \sqrt{\pi}}$ and Eq. 13 to obtain $\rho_{F}=$ $\rho_{B} \exp \left(-2 n\left|z-z^{\prime}\right|\right)$. Thus, in the thermodynamic limit, the short range correlations in a harmonic potential are identical to those of a periodic system. In the momentum distributions, this shows up as a Lorentzian tail, Eq. 18, with a width of $4 n$, where $n$ is the linear density. This feature is confirmed in the inset of Fig. 11 where the momentum is plotted on a logarithmic scale in units of the density $n$ to show how at large momentum all the distributions line up with a Lorentzian of width $4 n$ (solid line). This Lorentzian momentum tail will be a clear experimental signature of the strongly interacting regime discussed here.

In conclusion, duality in 1D many body systems provides a powerful method for understanding and calculating correlations in the strongly interacting limit. The system we consider, namely, strongly interacting spin-polarized 1D fermions with zero-range interactions, is dual to a noninteracting gas of bosons and provides an excellent prospect to study duality in actual experiments with ultracold atom gases. The exponentially decaying correlations described here will have a clear and measurable effect in these experiments and will hopefully spur on further theoretical inquiries as well.

This work was funded by the NSF under grant PHY0354882 .
[1] S. Tomonaga, Prog. Theor. Phys. 5, 544 (1950).

[2] J. M. Luttinger, J. Math Phys. 4, 1154 (1963).

[3] L. Tonks, Phys. Rev. 50, 955 (1936).

[4] M. D. Girardeau, J. Math Phys. 1, 516 (1960).

[5] S. Coleman, Phys. Rev. D 11, 2088 (1975).

[6] T. Cheon and T. Shigehara, Phys. Rev. Lett. 82, 2536 (1999).

[7] S. Albeverio, L. Dabrobski, and S. M. Fei, arXiv quant-ph/0001089 (2000).

[8] B. E. Granger and D. Blume, Phys. Rev. Lett. 92, 133202 (2004).

[9] M. D. Girardeau and M. Olshanii, arXiv cond-mat/0309396 (2003).

[10] H. Grosse, E. Langmann, and C. Paufler, arXiv math-ph/0401003 (2004).

[11] A. Görlitz et al., Phys. Rev. Lett. 87, 130402 (2001).

[12] S. Dettmer et al., Phys. Rev. Lett. 87, 160406 (2001).

[13] S. Richard et al., Phys. Rev. Lett. 91, 010405 (2003).

[14] B. Paredes et al., Nature 429, 277 (2004).

[15] T. Kinoshita, T. Wenger, and D. S. Weiss, Science 305, 1125 (2004).
[16] M. Olshanii, Phys. Rev. Lett. 81, 938 (1998).

[17] E. H. Lieb and W. Liniger, Phys. Rev. 130, 1605 (1963).

[18] J. B. McGuire, J. Math Phys. 5, 622 (1964).

[19] C. N. Yang and C. P. Yang, J. Math Phys. 10, 1115 (1969).

[20] S. Inouye et al., Nature 392, 151 (1998).

[21] H. G. Vaidya and C. A. Tracy, Phys. Rev. Lett. 42, 3 (1979).

[22] D. M. Gangardt and G. V. Shlyapnikov, Phys. Rev. Lett. 90, 010401 (2003).

[23] K. V. Kheruntsyan, D. M. Gangardt, P. D. Drummond, and G. V. Shlyapnikov, Phys. Rev. Lett. 91, 040403 (2003).

[24] M. Olshanii and V. Dunjko, Phys. Rev. Lett. 91, 090401 (2003).

[25] G. E. Astrakharchik and S. Giorgini, Phys. Rev. A 68, 031602 (2003).

[26] F. D. M. Haldane, J. Phys. C 14, 2585 (1981).

[27] K. Kanjilal and D. Blume, arXiv:physics/0406022 (2004).

[28] M. D. Girardeau, H. Nguyen, and M. Olshanii, arXiv cond-mat/0403721 (2004).

[29] C. A. Regal, C. Ticknor, J. L. Bohn, and D. S. Jin, Phys. Rev. Lett. 90, 053201 (2003). 